\title{
GARIMPO DO TUCANO: ANÁLISE AMBIENTAL DE UM GARIMPO ABANDONADO NO NORDESTE DE GOIÁS
}

\author{
GARIMPO DO TUCANO: ENVIRONMENTAL ANALYSIS OF AN ABANDONED \\ GOLD MINE IN NORTHEASTERN GOIÁS
}

\author{
GARIMPO DEL TUCANO: ANÁLISIS AMBIENTAL DE UNA MINA DE ORO \\ ABANDONADA AL NOROESTE DE GOIÁS
}

\author{
Luciana Gonçalves Tibiriçá - Universidade Federal de Goiás - Goiânia - Goiás - Brasil \\ andaluzita@gmail.com \\ Cláudia Valéria de Lima - Universidade Federal de Goiás - Goiânia - Goiás - Brasil \\ claudia@iesa.ufg.br
}

Nilson Francisquini Botelho - Universidade de Brasília - Brasília - Distrito Federal - Brasil

nilsonfb@unb.br

\section{Resumo}

A atividade garimpeira integra as atividades de mineração, contudo sua existência leva à ocorrência de impactos no ambiente, além de envolver a questão social de maneira mais evidente. Esta pesquisa investiga a questão garimpeira pela ótica de seus passivos ambientais, determinando a concentração de mercúrio nos sedimentos da barragem e na pilha de rejeitos de um garimpo localizado no município de Monte Alegre de Goiás (GO). Para a determinação da salubridade da área, foram realizadas análises multielementares de sedimentos e análises físico-químicas nas águas presentes no garimpo em questão. Os resultados indicam locais nos quais 0 monitoramento deve ser frequente e apontam uma área que deve ser isolada, dada a possibilidade de contaminação. Observa-se ainda a necessidade de realização de estudos nas áreas vizinhas com o objetivo de determinar se há condições ambientais similares.

Palavras-chave: mercúrio, garimpo, saúde humana.

\begin{abstract}
Mining activities have a large impact on the environment as well as the evident social issues. This research investigates the issue of mining through the lens of environmental liabilities, determining concentrations of mercury in sediment of dams and piles of debris in the location of the mine in Monte Alegre, Goiás-GO. To determine the quality of health in the area, multidimensional analyses were performed on the sediment and physical chemicals present in the water in the mining area in question. The results indicate the locations which should be frequently monitored because of the possibility of contamination, which should therefore be isolated. In addition, a need to conduct studies in neighboring areas was observed, in order to determine if there exists similar environmental impacts.
\end{abstract}

Keywords: mercury, mining, human health.

\section{Resumen}

La "minería artesanal" integra una las actividades de la minería, pero su existencia conlleva a la incidencia de impactos en el ambiente, además de que envuelvan la cuestión social de manera más evidente. Este estudio investiga la cuestión de la minería artesanal por la óptica de sus pasivos ambientales, determinando la concentración de mercurio en los sedimentos de la represa y en los residuos de desechos de una reserva de oro localizada en el municipio de Monte Alegre de Goiás-G0. Para determinar la salubridad del área, fueron realizados análisis multielementares de sedimentos y análisis físico-químicos en las aguas presentes en la reserva de 
oro en cuestión. Los resultados indican locales en los cuáles el seguimiento debe ser frecuente debido a la posibilidad de contaminación y apuntan a un área que debe ser aislada, dada la posibilidad de contaminación. Se observa aún la necesidad de realizar estudios en las áreas vecinas visando determinar se hay condiciones ambientales similares.

Palabras clave: mercurio, minería artesanal, salud humana.

Introdução

A mineração, atividade realizada pelo homem desde os primórdios da humanidade, com o domínio do fogo e a fabricação de armas, sempre exigiu a retirada de bens minerais da natureza. Sua existência sempre foi considerada um "mal necessário" e, de acordo com Mathis et al. (1997), por estar associada a fatores de crescimento econômico, acabou ganhando enfoque negativo. Entretanto, as formas de extração mineral e suas consequências começaram a ser discutidas a partir da "evolução" desencadeada com o desenvolvimento de novas tecnologias e o avanço das ciências. Mesmo se tratando de uma atividade necessária para o bem-estar da sociedade, que conta com o desenvolvimento de técnicas para minimizar os impactos ambientais causados, a mineração passou a ser considerada uma atividade poluidora e ameaçadora ao meio ambiente. No Brasil, é executada por empresas de grande e médio porte ou por pequenas empresas e garimpos. Os garimpos são observados com reservas, por envolverem a questão social de maneira mais evidente e serem carentes de apoio governamental e financeiro.

A questão garimpeira, nesta pesquisa, é analisada a partir de seu principal passivo ambiental: a possibilidade de contaminação do ambiente. Para verificar essa questão, foi determinada a concentração de mercúrio em sedimentos, rejeitos de garimpagem e na água.

\section{Garimpo do Tucano}

A área de estudo desta pesquisa é o Garimpo do Tucano, localizado no distrito de Riacho dos Cavalos, município de Monte Alegre de Goiás, distante 700 km de Goiânia, capital do Estado (Figura 1). Na região de Monte Alegre de Goiás, de acordo com Tibiriçá-Resende et al. (2003), ocorrem rochas metamórficas de idade proterozoica (com aproximadamente 1.500 milhões de anos) representadas por xistos grafitosos e rochas ígneas de mesma idade, representadas por granitos, os quais se encontram 
associados a pegmatitos e zonas hidrotermalizadas que permitiram a formação de turmalina e cassiterita. O ouro localiza-se em veios de quartzo encaixados entre xisto e granito. Outras unidades geológicas presentes são as rochas sedimentares do grupo Araí, responsáveis pelas porções mais elevadas da paisagem, e os calcários do grupo Bambuí, que ocorrem a leste da sede do município de Monte Alegre de Goiás.

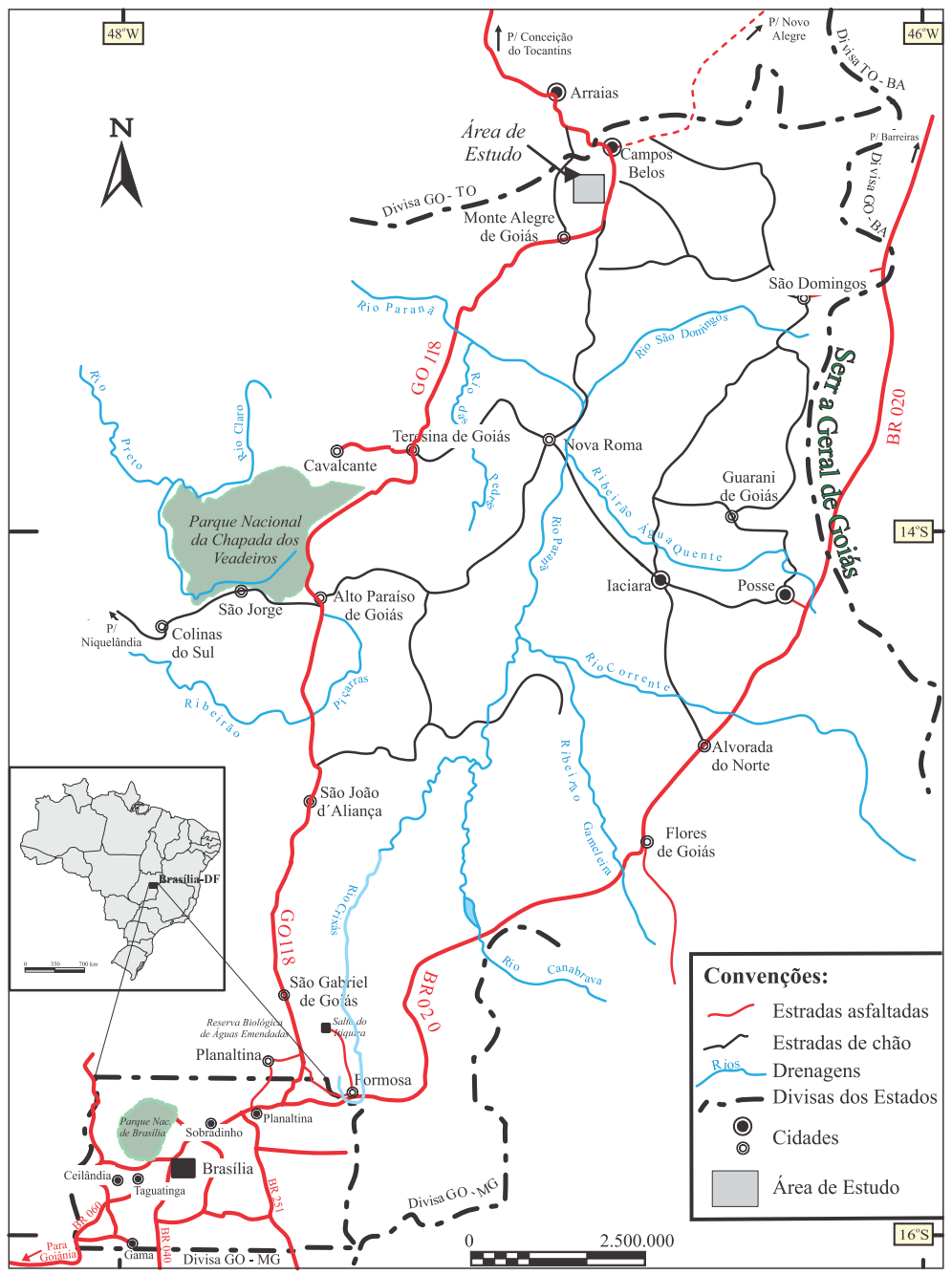

Figura 1 - Mapa de localização da área de estudo 
De acordo com Lazarin e Rabelo (1984), a ocupação do território de Monte Alegre de Goiás pelos garimpeiros deu-se na década de 1970, motivada pela mineração artesanal de ouro e cassiterita, mas sua história durou pouco mais de dez anos. A população local (cerca de 1/3), imigrante de Estados vizinhos (Maranhão, Bahia, Minas Gerais), e um considerável número de garimpeiros expulsos da Província Estanífera de Rondônia constituíram a maioria do contingente que se instalou pelos vários garimpos. Atualmente, os garimpos de cassiterita estão desativados devido ao baixo valor do minério no mercado. Os garimpos de ouro estão, em sua maioria, desativados, e os que estão em atividade o fazem de maneira bastante precária, artesanal. Atualmente, o Garimpo do Tucano encontra-se inativo e no local restaram áreas de extração e descarte de estéreis abandonados: a pilha de rejeitos, uma lagoa onde originalmente estava a cava do garimpo e uma barragem. Na área, há uma chácara habitada por uma família.

\section{Principais aspectos do mercúrio}

De acordo com Figueiredo (2000), a contaminação por mercúrio dá-se pela exposição ocupacional, que ocorre tradicionalmente relacionada às atividades garimpeiras (absorção direta, inalação e ingestão de água), ou pela exposição não ocupacional, que pode ocorrer por meio da alimentação. Ambas podem conduzir a efeitos adversos na saúde humana e a desastres ambientais. Nesta pesquisa, foram consideradas as possibilidades de exposições ambientais, já que, por estar o Garimpo do Tucano inativo e praticamente abandonado, inviabiliza o levantamento de atividades ocupacionais.

Análises preliminares realizadas no ano de 1999 (Tibiriçá-Resende et al., 2003), em amostras da pilha de rejeitos, confirmam que nelas há a presença de mercúrio (Tabela 1). Contrapondo estes valores aos encontrados nas amostras de análises realizadas por Thornton (1996), conforme Tabela 2, obtêm-se valores de referência que ratificam a contaminação da área estudada. 
Tabela 1 - Resultados preliminares de contaminação mercurial de sedimentos da barragem e das pilhas de rejeitos

\begin{tabular}{|c|c|c|}
\hline Amostra & Local & PPM \\
\hline $1 \mathrm{a}$ & Pilha de rejeito & 10,17 \\
\hline $9 \mathrm{a}$ & Pilha de rejeito & 4,18 \\
\hline Amostra & Local & PPM \\
\hline $6 \mathrm{a}$ & Sedimento da barragem & 0,1 \\
\hline $7 \mathrm{a}$ & Sedimento da barragem & 0,06 \\
\hline $8 \mathrm{a}$ & Sedimento da barragem & 0,08 \\
\hline $10 \mathrm{a}$ & Sedimento da barragem & 0,04 \\
\hline
\end{tabular}

Fonte: Tibiriçá-Resende et al. (2003)

Tabela 2 - Concentrações de $\mathrm{Hg}_{\mathrm{T}}$ em amostras de garimpo de ouro do Rio Tapajós (1996)

\begin{tabular}{|c|c|c|c|c|}
\hline Categoria (ppm) & Cuiu Cuiu & Jacareacanga & Itaituba & Crepori \\
\hline Sedimento de corrente & 1,1 & 0,34 & 0,28 & 0,97 \\
\hline Rejeito & 31,0 & 11,0 & 150 & 210 \\
\hline
\end{tabular}

\section{Operacionalização da pesquisa}

A operacionalização desta pesquisa segue procedimentos já desenvolvidos e reconhecidos, visando à interpretação da condição geoambiental da área do Garimpo do Tucano de maneira integrada (sedimentos, rejeitos e água), para alcançar os objetivos principais: determinar as áreas consideradas de risco à saúde humana, buscando a proteção da comunidade vizinha e dos recursos naturais remanescentes, e apontar os locais onde a ocupação e o uso do solo devem ser controlados ou mesmo inibidos.

Os sedimentos da barragem coletados - na barragem e na cava do garimpo - foram analisados no Laboratório de Química Analítica e Ambiental do Instituto de Química da Universidade de Brasília. As amostras foram peneiradas na fração argila, em uma granulometria de 400 mesh (38 $\mu \mathrm{m})$, e analisadas pela técnica de Espectroscopia de Absorção Atômica com geração de Vapor Frio - EAA-VP, por meio da redução dos íons $\mathrm{Hg}^{2+}$ contidos em $1 \mathrm{ml}$ de amostra digerida, diluída em $10 \mathrm{ml}$ de $\mathrm{NaCl}$ 1\% com 2,5\% de uma solução redutora, após geração de curva analítica na faixa de 1 a 20 ppb de mercúrio.

As amostras de água foram encaminhadas para o laboratório ACME, no Canadá, onde foi realizada a identificação multielementar das águas por ICP-MS. Foram coletadas três amostras ao longo das margens da 
barragem e duas amostras nas margens da cava do garimpo, e para todas elas foram analisados todos os elementos. As coletas de água se deram na interface água-ar, exclusivamente no Garimpo do Tucano, e foram escolhidos três pontos para análise de dados físico-químicos e amostragem na barragem, e dois pontos de coleta de dados físico-químicos e um de amostragem na cava. Os valores físico-químicos foram obtidos por meio do equipamento analítico de qualidade de água multiparâmetro, denominado Horiba, utilizado para os dados de $\mathrm{pH}$, temperatura, salinidade, ORP, condutividade e oxigênio dissolvido.

\section{Índice de Geoacumulação}

Para Moreira e Boaventura (2003), o Índice de Geoacumulação ( $\mathrm{I}_{\text {geo }}$ ) proposto por Muller permite a avaliação da contaminação do sedimento por meio da relação entre os teores de metais encontrados na região estudada e um valor referencial equivalente à média mundial para metais associados às argilas. $\mathrm{O}$ valor obtido permite classificar os níveis de metais em sete extratos, com intensidades progressivas de contaminação, conforme exposto na Tabela 3.

Tabela 3 - Classificação, grau e classe do I

\begin{tabular}{|l|c|c|}
\hline \multicolumn{1}{|c|}{ Classificação } & Classe do I Ieo $_{\text {geo }}$ & Grau do I $_{\text {geo }}$ \\
\hline Extremamente poluído & 6 & $>5$ \\
\hline Fortemente a extremamente poluído & 5 & $4 \mathrm{a} \leq 5$ \\
\hline Fortemente poluído & 4 & $>3 \mathrm{a} \leq 4$ \\
\hline Moderadamente a fortemente poluído & 3 & $>2 \mathrm{a} \leq 3$ \\
\hline Moderadamente poluído & 2 & $1 \mathrm{a} \leq 2$ \\
\hline Não poluído a moderadamente poluído & 1 & $>0$ a $\leq 1$ \\
\hline Praticamente não poluído & 0 & $<0$ \\
\hline
\end{tabular}

Fonte: Adaptado de Moreira e Boaventura (2003) e Luiz-Silva et al. (2002)

Cada uma das amostras, inclusive as coletadas em 1999, teve seu valor calculado de acordo com a fórmula abaixo:

$\mathrm{I}_{\text {geo }}=\log 2\left(\mathrm{C}_{\mathrm{N}} / 1,5 \mathrm{C}_{\mathrm{B}}\right) \quad$, onde

$\mathrm{C}_{\mathrm{N}}=$ concentração do elemento " $\mathrm{n}$ " na fração argila do sedimento a ser classificado; 
$\mathrm{C}_{\mathrm{B}}=$ concentração média de "background" para argilas na crosta terrestre (folhelho médio padrão), que é 0,18;

1,5 = fator de correção para possíveis variações do "background" causado por diferenças litológicas.

Resultados e discussões

Para operacionalizar a realização deste trabalho, dividiu-se o Garimpo do Tucano em quatro unidades (Figura 2):

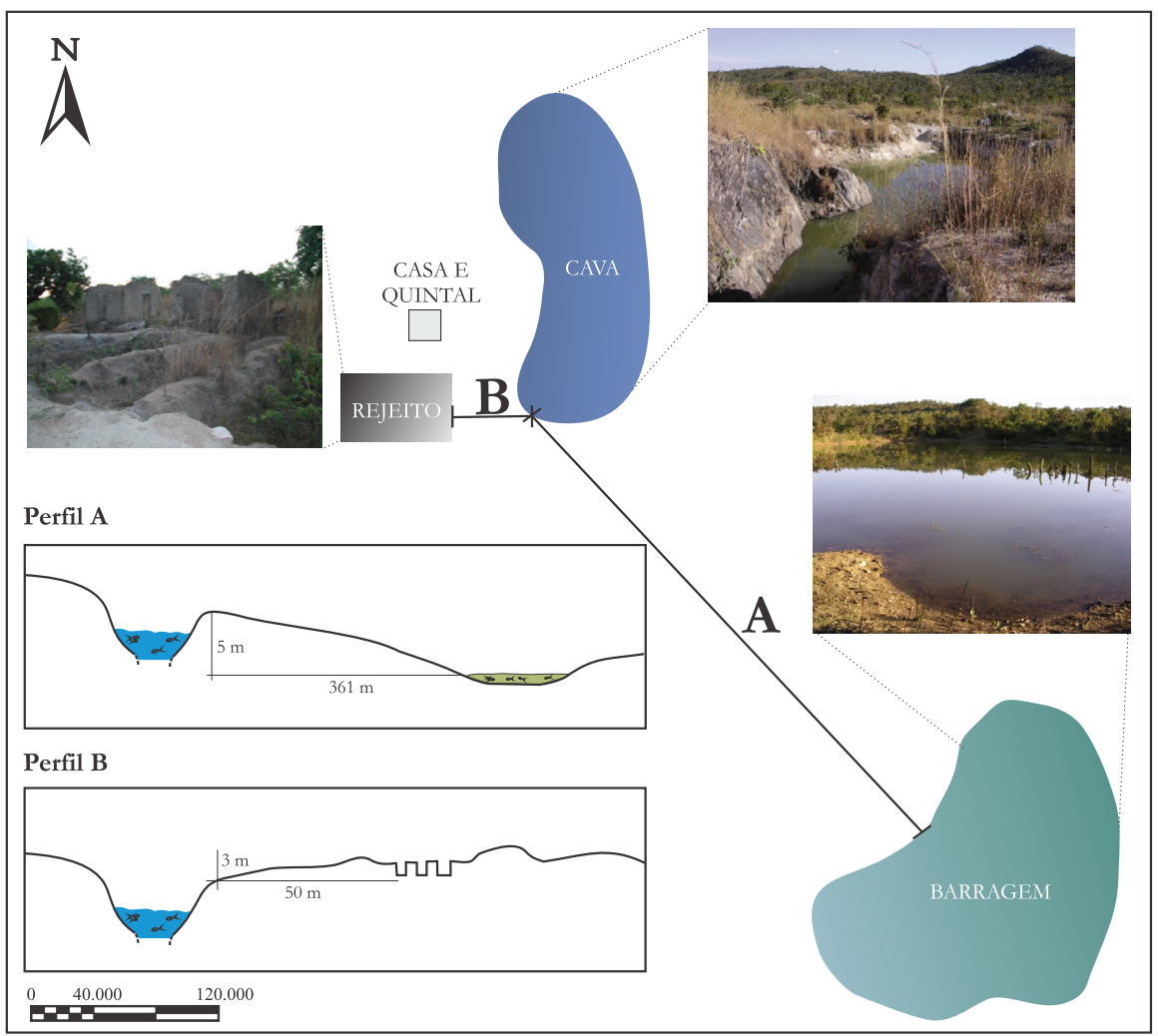

Figura 2 - Esquema de localização das quatro unidades do Garimpo do Tucano 
- barragem: utilizada para diversão da comunidade, dessedentação animal e criação de peixes;

- área onde foi depositada a pilha de rejeitos: onde era lavado o material e separado o mercúrio do ouro;

- casa e quintal da chácara;

- cava do garimpo: esta área é utilizada atualmente para a lavagem de roupas e louças e para diversão da comunidade.

Entre os impactos ambientais negativos observados em praticamente todas as atividades garimpeiras, e que na área estudada não se apresentaram diferentes, estão o impacto visual, a destruição da vegetação nativa e o armazenamento inadequado dos rejeitos lavrados de ouro, permitindo contato direto com moradores e com o ecossistema. Na barragem localizada abaixo da área garimpada são criados peixes, utilizados pelos moradores, eventualmente, para alimentação.

Resultados das análises

A. Sedimentos da barragem e das pilhas de rejeitos

Os teores de mercúrio encontrados nas amostras analisadas estão na tabela abaixo:

Tabela 4 - Concentrações de $\mathrm{Hg}_{\mathrm{T}}$ encontrados nos sedimentos e rejeitos do Garimpo do Tucano

\begin{tabular}{|l|c|c|c|c|c|l|}
\hline \multirow{2}{*}{ Amostra } & \multirow{2}{*}{$\begin{array}{c}\text { Ano de } \\
\text { coleta }\end{array}$} & \multicolumn{3}{|c|}{ Concentração de mercúrio (ppm) } & \multirow{2}{*}{ Localização } \\
\cline { 3 - 6 } & Média & Desvio-padrão & Máx. & Mín. & \\
\hline LT TUC 1 & 2004 & 0,273 & 0,021 & 0,290 & 0,250 & Barragem \\
\hline LT TUC 2 & 2004 & 0,132 & 0,005 & 0,126 & 0,135 & Barragem \\
\hline LT TUC 3 & 2004 & 0,193 & 0,010 & 0,200 & 0,186 & Barragem \\
\hline LT TUC 4 & 2005 & 1,310 & 0,113 & 1,380 & 1,180 & Pilha de rejeito \\
\hline LT TUC 5 & 2005 & 21,050 & 0,069 & 21,090 & 20,970 & Pilha de rejeito \\
\hline LT TUC 6 & 2005 & 0,338 & - & - & - & Barragem \\
\hline LT TUC 7 & 2005 & 0,299 & 0,021 & 0,313 & 0,284 & Barragem \\
\hline LT TUC 8 & 2005 & 0,153 & 0,004 & 0,158 & 0,151 & Barragem \\
\hline LT TUC 9 & 2005 & 0,291 & 0,015 & 0,305 & 0,276 & Barragem \\
\hline
\end{tabular}


O I ${ }_{\text {geo }}$ para o Garimpo do Tucano é apresentado na Tabela 5 e na Figura 3. O mapa foi elaborado nos softwares ARCVIEW e ARCGIS versão 9.0, utilizando-se os métodos de Krigagem e Prediction Map. Elaborou-se este mapa no intuito de se ter ciência da discrepância entre os dados encontrados na pilha de rejeitos e na barragem, o que pode ser observado a partir dos pontos ao Norte, indicando a localização da barragem, e dos pontos ao Sul, marcando a posição da pilha de rejeitos. Os pontos de exposição, ou seja, aqueles pontos onde há potencial contato com o mercúrio, também podem ser observados na Figura 3.

Tabela 5 - Índice de Geoacumulação $\left(I_{\text {geo }}\right)$ do Garimpo do Tucano, calculado a partir dos valores médios de concentração de $\mathrm{Hg}_{\mathrm{T}}$

a) Barragem

\begin{tabular}{|c|c|c|c|c|}
\hline Amostra & Ano de coleta & $\mathbf{H g}_{\mathrm{T}}(\mathbf{p p m})$ & $\mathbf{I}_{\text {geo }}$ & Classe \\
\hline 6 a & 1999 & 0,1 & $-1,433$ & 0 \\
\hline $7 \mathrm{a}$ & 1999 & 0,06 & $-2,170$ & 0 \\
\hline 8 a & 1999 & 0,08 & $-1,755$ & 0 \\
\hline 10 a & 1999 & 0,04 & $-2,755$ & 0 \\
\hline LT TUC 1 & 2004 & 0,273 & 0,015 & 1 \\
\hline LT TUC 2 & 2004 & 0,132 & $-1,032$ & 0 \\
\hline LT TUC 3 & 2004 & 0,193 & $-0,484$ & 1 \\
\hline LT TUC 6 & 2005 & 0,338 & 0,324 & 1 \\
\hline LT TUC 7 & 2005 & 0,299 & 0,147 & 0 \\
\hline LT TUC 8 & 2005 & 0,153 & $-0,819$ & 1 \\
\hline LT TUC 9 & 2005 & 0,291 & 0,108 & 0 \\
\hline
\end{tabular}

b) Pilha de rejeitos

\begin{tabular}{|c|c|c|c|c|}
\hline Amostra & Ano de coleta & $\mathbf{H g}_{\mathrm{T}} \mathbf{( p p m )}$ & $\mathbf{I}_{\text {geo }}$ & Classe \\
\hline 1 a & 1999 & 10,17 & 5,235 & 6 \\
\hline 9 a & 1999 & 4,18 & 3,95 & 4 \\
\hline LT TUC 4 & 2005 & 1,310 & 2,278 & 3 \\
\hline LT TUC 5 & 2005 & 21,050 & 6,285 & 6 \\
\hline
\end{tabular}




\section{Mapa de Índice de Geoacumulação GARIMPO DO TUCANO}

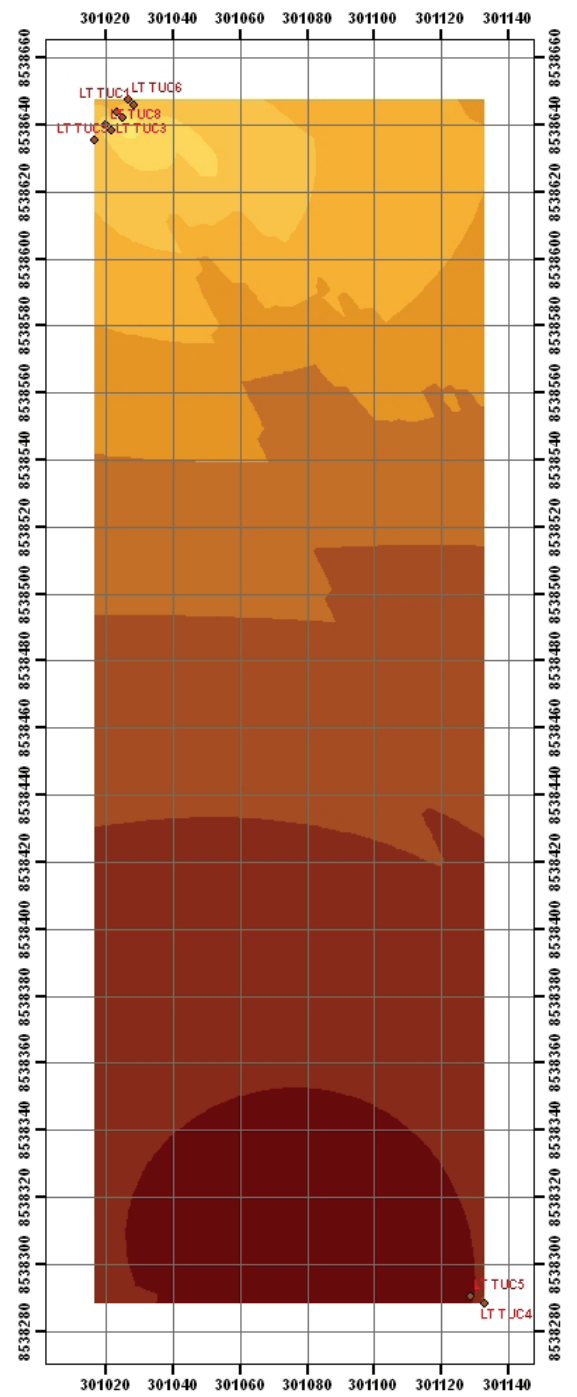

\section{LEGENDA}

- pontos de amostragem

\section{CLASSES DE Igeo KRIGING - Prediction Map}
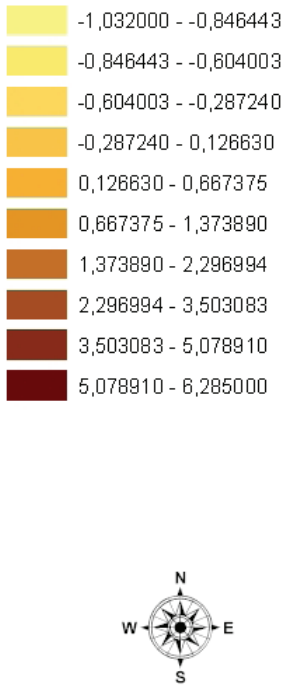

SISTEMADE COORDENADAS UTM DATUM HORIZONTAL WGS-84 MERIDIANO CENTRAL $51 \% \mathrm{WG}$

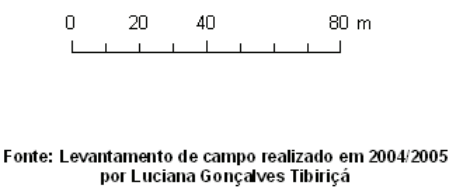

Mapa elaborado por Tibiriçá em 2006.

Figura 3 - Espacialização dos pontos de amostragem e dos valores de $\mathrm{I}_{\text {geo }}$ para o Garimpo do Tucano 


\section{B. Água}

Os resultados obtidos nas análises dos parâmetros físico-químicos da água encontram-se na Tabela 6 . O valor de $\mathrm{pH}$ em torno de 5,3, encontrado em ambos os locais de amostragem, potencializa, segundo Baird (2002), a metilação do mercúrio. Para Parey (1993), em águas com valores de $\mathrm{pH}$ abaixo de 5,5 (encontrados em todas as amostragens) a maior parte dos pequenos organismos é prejudicada ou morta.

Tabela 6 - Valores físico-químicos encontrados nas águas da barragem e da cava

a) Barragem

\begin{tabular}{|c|c|c|c|c|c|c|}
\hline Ponto & $\mathbf{p H}$ & $\begin{array}{c}\text { Condutividade } \\
(\mathbf{m} \text { S/s) }\end{array}$ & $\begin{array}{c}\text { TDS } \\
\mathbf{( g / L )}\end{array}$ & $\begin{array}{c}\text { Turbidez } \\
(\mathbf{g} / \mathbf{L})\end{array}$ & $\begin{array}{c}\text { Temperatura } \\
\left({ }^{\mathbf{}} \mathbf{C}\right)\end{array}$ & $\begin{array}{c}\text { Potencial } \\
\text { Redox (mV) }\end{array}$ \\
\hline 1 & 5,16 & 15,8 & 0,12 & -10 & 25,10 & N M \\
\hline 2 & 5,45 & 13 & 0,10 & 58 & 25,29 & N M \\
\hline 3 & 5,3 & 11,7 & 0,09 & -10 & 25,21 & 235 \\
\hline
\end{tabular}

b) Cava

\begin{tabular}{|c|c|c|c|c|c|c|}
\hline Ponto & $\mathbf{p H}$ & $\begin{array}{c}\text { Condutividade } \\
(\mathbf{m ~ S / s})\end{array}$ & $\begin{array}{c}\text { TDS } \\
(\mathbf{g} / \mathbf{L})\end{array}$ & $\begin{array}{c}\text { Turbidez } \\
\mathbf{( g / L )}\end{array}$ & $\begin{array}{c}\text { Temperatura } \\
\left({ }^{\mathbf{}} \mathbf{C}\right)\end{array}$ & $\begin{array}{c}\text { Potencial } \\
\text { Redox }(\mathbf{m V})\end{array}$ \\
\hline 1 & 5,19 & 20,0 & 0,13 & -1 & 26,82 & 245 \\
\hline 2 & 5,35 & 18,2 & 0,12 & 47 & 26,99 & 242 \\
\hline
\end{tabular}

Os valores encontrados, apresentados acima, corroboram a fonte da água proveniente de granitos, de acordo com Parey (1993) e com os dados obtidos por Tibiriçá-Resende et al. (1999). Esteves (1988) esclarece que os íons mais diretamente responsáveis pelos valores de condutividade elétrica em águas interiores são os macronutrientes (cálcio, magnésio, potássio, sódio, carbonato, sulfato e cloreto), mas que os valores de condutividade estão mais relacionados com as características geoquímicas da região e com as condições climáticas.

O resultado apresentado para o Potencial Redox indica ambiente oxidante tanto na barragem quanto na cava do garimpo, e os valores de pH implicam águas ácidas. Já os valores encontrados para a temperatura dos pontos de amostragem estão pouco acima da considerada ideal; entretanto, dada a localização geográfica da área, os valores são coerentes.

Os vários elementos químicos analisados tiveram seus valores distribuídos na Tabela 7. Alguns elementos obtiveram resultados inferiores 
ao limite de detecção (LD) do aparelho, significando que eles são encontrados em quantidades mínimas, até porque são elementos-traço de rochas e raramente encontrados em abundância no ambiente. Entre eles estão o mercúrio e o enxofre. O valor obtido para o mercúrio era esperado, posto que este elemento raramente é encontrado dissolvido na água. Entretanto, outros elementos mostraram-se importantes para a análise ambiental e são discutidos na tabela abaixo. Na Tabela 7a estão os elementos cujos valores são semelhantes nos dois pontos de amostragem: a barragem e a cava do garimpo. Já os resultados da Tabela 7b apresentam-se díspares nos dois pontos, indicando uma disparidade química na composição das águas.

Dos elementos da Tabela 7a, cujos valores são semelhantes nos dois pontos de amostragem, apenas o Molibdênio (Mo) e o Potássio (K) são importantes para as plantas. Entretanto, os valores de Arsênio (As), Boro (B), Bário (Ba), Molibdênio (Mo) e Antimônio (Sb) são inferiores ao padrão. Já os valores de Hólmio (Ho), Índio (In), Potássio (K), Lutécio (Lu), Escândio (Sc), Térbio (Tb), Túlio (Tm) e Itérbio (Yb) são superiores ao estabelecido no padrão. Este fato pode ser explicado pela geoquímica das rochas locais.

$\mathrm{Na}$ Tabela 7b estão os valores dos outros elementos químicos que apresentaram valores díspares entre os dois pontos de amostragem, indicando se tratar de ambientes diferentes. Essa diversidade pode ser observada nos valores dos elementos considerados importantes para o ecossistema, principalmente o Alumínio (Al), Cloro (Cl), Cobalto (Co), Cromo (Cr), Cobre (Cu), Ferro (Fe), Fósforo (P), Manganês (Mn), Níquel (Ni), Chumbo (Pb), Sílica (Si), Estanho (Sn), Vanádio (V) e Zinco (Zn). Os valores encontrados para estes elementos são muito mais elevados na barragem, onde a temperatura é menor. Os valores de Cloro (Cl), Cálcio (Ca), Magnésio (Ma) e Sódio (Na) encontrados na cava do garimpo, onde a temperatura é superior em um grau, são mais elevados que os da barragem, principalmente devido à atividade humana, posto que a cava é utilizada para ações como lavar roupas e louças. O uso de sabão e detergente potencializa os valores destes elementos. Os valores de Potássio (K), Boro (B) e Molibdênio (Mo) são semelhantes nas duas localidades. 
Tabela 7 - Presença elementar nas águas da barragem e da cava a) valores elementares semelhantes nos dois pontos de amostragem

\begin{tabular}{|c|c|c|c|c|c|}
\hline \multirow{2}{*}{ Elemento } & \multirow{2}{*}{ Unidade } & \multicolumn{3}{|c|}{ Barragem } & Cava \\
\cline { 3 - 5 } & & LT-TUC 1 & LT-TUC 2 & LT-TUC 3 & LT-TUC 4 \\
\hline $\mathrm{B}$ & $\mathrm{ppb}$ & 9 & 9 & 9 & 7 \\
\hline $\mathrm{K}$ & $\mathrm{ppb}$ & 4476 & 4426 & 4800 & 4138 \\
\hline $\mathrm{Mo}$ & $\mathrm{ppb}$ & 0,2 & 0,1 & 0,1 & 0,2 \\
\hline $\mathrm{As}$ & $\mathrm{ppb}$ & 5,3 & 4,9 & 5,1 & 4 \\
\hline $\mathrm{Ba}$ & $\mathrm{ppb}$ & 43,81 & 41,69 & 46,71 & 37,75 \\
\hline $\mathrm{Ho}$ & $\mathrm{ppb}$ & 0,07 & 0,07 & 0,08 & $<0,01$ \\
\hline $\mathrm{In}$ & $\mathrm{ppb}$ & 0,01 & 0,01 & 0,01 & $<0,01$ \\
\hline $\mathrm{Lu}$ & $\mathrm{ppb}$ & 0,02 & 0,02 & 0,02 & $<0,01$ \\
\hline $\mathrm{Sb}$ & $\mathrm{ppb}$ & 0,14 & 0,07 & 0,07 & $<0,05$ \\
\hline $\mathrm{Sc}$ & $\mathrm{ppb}$ & 1 & 1 & $<1$ & $<1$ \\
\hline $\mathrm{Tb}$ & $\mathrm{ppb}$ & 0,1 & 0,09 & 0,1 & $<0,01$ \\
\hline $\mathrm{Tm}$ & $\mathrm{ppb}$ & 0,02 & 0,02 & 0,02 & $<0,01$ \\
\hline $\mathrm{Yb}$ & $\mathrm{ppb}$ & 0,16 & 0,14 & 0,15 & $<0,01$ \\
\hline
\end{tabular}

b) valores elementares díspares nos dois pontos de amostragem

\begin{tabular}{|c|c|c|c|c|c|}
\hline \multirow{2}{*}{ Elemento } & \multirow{2}{*}{ Unidade } & \multicolumn{3}{|c|}{ Barragem } & Cava \\
\cline { 3 - 5 } & & LT-TUC 1 & LT-TUC 2 & LT-TUC 3 & LT-TUC 4 \\
\hline $\mathrm{Al}$ & $\mathrm{ppb}$ & 22010 & 21839 & 23960 & 139 \\
\hline $\mathrm{Ca}$ & $\mathrm{ppb}$ & 917 & 867 & 881 & 3693 \\
\hline $\mathrm{Cl}$ & $\mathrm{ppm}$ & 1 & 1 & $<1$ & 5 \\
\hline $\mathrm{Co}$ & $\mathrm{ppb}$ & 1,21 & 1,14 & 1,27 & 0,1 \\
\hline $\mathrm{Cr}$ & $\mathrm{ppb}$ & 7,9 & 7,3 & 8,1 & 1,2 \\
\hline $\mathrm{Cu}$ & $\mathrm{ppb}$ & 3,9 & 3,8 & 3,8 & 0,7 \\
\hline $\mathrm{Fe}$ & $\mathrm{ppb}$ & 7533 & 7001 & 7594 & 30 \\
\hline $\mathrm{Mg}$ & $\mathrm{ppb}$ & 643 & 663 & 657 & 2065 \\
\hline $\mathrm{Mn}$ & $\mathrm{ppb}$ & 44,98 & 42,51 & 48,27 & 3,11 \\
\hline $\mathrm{Na}$ & $\mathrm{ppb}$ & 5440 & 5515 & 5591 & 7826 \\
\hline $\mathrm{P}$ & $\mathrm{ppb}$ & 244 & 242 & 224 & 27 \\
\hline $\mathrm{Si}$ & $\mathrm{ppb}$ & 40280 & 38369 & 41218 & 3453 \\
\hline $\mathrm{Sn}$ & $\mathrm{ppb}$ & 5,67 & 5,42 & 5,93 & $<0,05$ \\
\hline $\mathrm{V}$ & $\mathrm{ppb}$ & 5,9 & 5,3 & 5,9 & $<0,2$ \\
\hline $\mathrm{Zn}$ & $\mathrm{ppb}$ & 20 & 15,9 & 17 & 0,7 \\
\hline
\end{tabular}


A distância entre a cava do garimpo e a barragem é de 360 metros aproximadamente, com desnível de 5 metros, como foi apresentado na Figura 3. Na cava do garimpo há contato de duas litologias diferentes (denominadas "Granito $\gamma$ 2" e "Formação Ticunzal"). Na região onde se localiza a barragem estão os sedimentos oriundos do Granito $\gamma 2$ e estes estão mais distanciados do contato com os xistos da Formação Ticunzal, apesar de haver na formação geológica sedimentos também desta fonte. Este fato é o principal responsável pelas diferenciações encontradas nas análises das águas destes dois pontos. Comparando-se os resultados encontrados nas análises de água com a geologia da área e a geoquímica das rochas desta localidade realizada por Tibiriçá et al. (1999), explica-se a presença em maior abundância de alguns elementos essenciais, como Cálcio e Sódio, nas águas da cava do garimpo.

\section{Considerações finais}

Segundo os dados encontrados nas amostras e apresentados anteriormente, a área do Garimpo do Tucano possui contaminação mercurial considerável na pilha de rejeitos e em menor quantidade nos sedimentos da barragem, com considerável possibilidade de dispersão da contaminação por toda a área, conforme apresentado pelo Índice de Geoacumulação $\left(\mathrm{I}_{\text {geo }}\right.$. Entretanto, essa contaminação, aparentemente, parece limitar-se ao mercúrio inorgânico, pois o Potencial Redox encontrado indica ambiente oxidante, inviabilizando a metilação, fato corroborado ainda pelos teores de enxofre na água, que se encontram abaixo do limite de detecção do aparelho. Consideram-se ainda fatores favoráveis à não propagação do mercúrio no sistema o tempo relativamente curto de existência do garimpo - pouco mais de cinco anos - e a baixa declividade do terreno dificultando a dispersão dos resíduos desse garimpo.

As análises de água realizadas na barragem e na cava foram conclusivas quanto à inexistência de contaminação por mercúrio, corroborando as análises realizadas nos sedimentos da barragem. Assim, conclui-se que as reações químicas do Ciclo do Mercúrio não ocorrem no ambiente da barragem, especialmente em decorrência de o ambiente oxidante não ser favorável à metilação do mercúrio.

O fato de o garimpo estar desativado há muitos anos faz com que a emissão atmosférica do gás não exista. O depósito do metal está em uma 
área a montante da barragem, fato comprovado pelas análises dos sedimentos - o que pode levar a uma eventual contaminação, principalmente via lençol freático, ou caso haja perda de solo ou remobilização deste. Outra fonte provável pode ser a ocorrência de queimadas, o que disponibilizaria o metal diretamente dentro do ciclo já apresentado. Entretanto, por tratar-se de uma área onde não há atividade agropastoril, a queimada passa a ser um evento com causas naturais e de frequência indeterminada. Mesmo em caso de haver a remobilização do metal, seria necessária a incorporação do ambiente aquático no processo, já que as condições de pH são favoráveis à metilação desse metal.

Com o estabelecimento dos pontos, pelo Índice de Geoacumulação $\left(\mathrm{I}_{\text {geo }}\right)$, onde há contaminação por mercúrio no Garimpo do Tucano fica clara a necessidade de isolar a área da pilha de rejeitos, para não haver o contato do homem e de animais. Também, verifica-se a impossibilidade de utilização desta área para o plantio de qualquer vegetal que possa ser ingerido, evitando assim a intoxicação por mercúrio.

Outra necessidade é a de realizar investigações em áreas similares da mesma região, pois verificou-se uma série de cavas em situação análoga à apresentada neste trabalho, o que pode maximizar o problema da potencial contaminação por mercúrio no município de Monte Alegre de Goiás.

\section{Agradecimentos}

Os autores agradecem à comunidade local pela sempre gentil colaboração nas etapas do trabalho de campo, e ao Instituto de Química da Universidade de Brasília pela parceria na realização das análises.

\section{Referências}

BAIRD, C. Química ambiental. 2. ed. Porto Alegre: Bookman, 2002. 622p.

ESTEVES, F. de A. Fundamentos de Limnologia. Rio de Janeiro: Interciência/ FINEP, 1988. 575p.

FIGUEIREDO, B.R. Minérios e ambiente. Campinas (SP): Editora UNICAMP, 2000. 401p.

LAZARIN, M. A.; RABELO, F. C. Garimpeiros do Nordeste de Goiás. In: ROCHA, G. A. (Org.). Em busca do ouro: garimpos e garimpeiros no Brasil. Rio de Janeiro: Marco Zero, 1984. p. 107-120. 
LUIZ-SILVA, W.; MATOS, R. H. R.; KRITOSCH, G. C. Geoquímica e Índice de Geoacumulação de Hg em sedimentos de superfície do estuário de Santos-Cubatão (SP). Química Nova, v. 25, n. 5, 2002.

MATHIS, A.; BRITO, D. C. de; BRUSEKE, F. J. Riqueza volátil: a mineração de ouro na Amazônia. Belém: Editora CEJUP, 1997. 290p.

MOREIRA, R. C. A.; BOAVENTURA, G. R. Referência geoquímica regional para a interpretação das concentrações de elementos químicos nos sedimentos da bacia do Lago Paranoá (DF). Química Nova, v. 26, n. 6, 2003.

PAREY, V. P. Manuais para gerenciamento de recursos hídricos. DVWK, 227/1993. Relevância de parâmetros de qualidade das águas aplicados às águas correntes. Parte I. Florianópolis: FATMA /DVWK, 1993. 103p.

THORNTON, I. Impacts of mining on the environment; some local, regional and global issues. Applied Geochemistry, v. 11, 1996.

TIBIRIÇÁ-RESENDE，L. G.; CÂNDIDO，L. W.; ALVARENGA，C. J. S. de; GUIMARÃES, J. E.; DARDENNE, M. A.; MOURA, M. A.; BOTELHO, N. F.; MENESES, P. R.; SANTOS, R. V. Projeto Monte Alegre de Goiás - subárea VIII - Riacho dos Cavalos. Trabalho final de graduação - Instituto de Geociências, Universidade de Brasília, Brasília, 1999.

TIBIRIÇÁ-RESENDE, L. G.; CÂNDIDO, L. W.; ALVARENGA, C. J. S. de; GUIMARÃES, J. E.; DARDENNE, M. A.; MOURA, M. A.; BOTELHO, N. F.; MENESES, P. R.; SANTOS, R. V. Análise preliminar dos impactos ambientais dos garimpos de cassiterita e ouro da região de Monte Alegre de Goiás (GO). In: VIII Simpósio de Geologia do Centro-Oeste, 2003. Anais... Cuiabá, 2003.

Luciana Gonçalves Tibiriçá - Graduada em Geologia pela Universidade de Brasília - Mestre em Geografia pela Universidade Federal de Goiás - Doutoranda em Geologia na Universidade de Brasília - Professora Assistente da Universidade Federal de Goiás.

Cláudia Valéria de Lima - Graduada em Geologia pela Universidade de Brasília - Mestre em Geociências pela Universidade Estadual de Campinas - Doutora em Geologia pela Universidade de Brasília - Professora Associada da Universidade Federal de Goiás.

Nilson Francisquini Botelho - Graduado em Geologia pela Universidade de Brasília - Mestre em Geologia pela Universidade de Brasília - Doutor em Geologia pela Université de Paris VI (Pierre et Marie Curie) - Professor Titular da Universidade de Brasília.

Recebido para publicação em 30 de julho de 2013 Aceito para publicação em 1 de novembro de 2013 